\title{
Frequency and implications of ambulatory surgery without a patient escort
}

\author{
[La fréquence et les implications de la chirurgie ambulatoire de patients non \\ accompagnés]
}

Frances Chung FrCPC, ${ }^{*}$ Ngozi Imasogie FrCA, ${ }^{*}$ Joyce Ho, ${ }^{*}$ Xiangqun Ning MD, ${ }^{*}$ Atul Prabhu FrCA, Bruna Curti RN†

Purpose: A study was undertaken to identify the characteristics and outcomes of ambulatory surgical patients without an escort.

Clinical features: During a 38-month period, the incidence of patients without an escort at one tertiary care institution was $0.2 \%(60 / 28,391)$. Five patients had their surgery cancelled. The other 55 patients had their surgery performed. Two groups of patients without an escort were identified: patients who were known not to have an escort preoperatively, and patients with "no show" escort. The number of patients with no show escort is much higher than the number of patients who were known not to have an escort preoperatively. The outcome of the no escort patients was compared with the matched control group of patients with an escort. There were no differences in the rates of unanticipated admission, emergency visits or readmission into the hospital within 30 days comparing the group of patients with and without an escort.

Conclusions: The absence of an escort in ambulatory surgical patients occurs in $0.2 \%$ of surgeries, and varies according to the type of service. The number of patients with no show escort is higher than the number of patients with known no escort preoperatively.

Objectif: Déterminer les caractéristiques et les suites de la chirurgie ambulatoire chez des patients sans accompagnateur.

Éléments cliniques : Pendant une période de 38 mois, l'incidence de patients non accompagnés qui se sont présentés à une institution de soins tertiaires a été de 0,2\% (60/28,39l). L'opération chirurgicale a été annulée pour cinq patients. Les 55 autres patients ont été opérés. Deux groupes de patients non accompagnés ont été recensés : ceux dont on savait avant l'opération qu'ils seraient seuls et ceux dont l'accompagnateur ne s'est pas présenté.
Ces derniers étaient plus nombreux que les premiers. L'évolution des patients sans accompagnateur a été comparée avec le groupe de patients témoins appariés qui étaient accompagnés. II n’y a pas eu de différence quant au taux d'admission hospitalière imprévue, aux visites à l'urgence ou à la réadmission à l'hôpital dans les 30 jours, entre les patients accompagnés ou non.

Conclusion: L'absence d'accompagnateur pour les patients de chirurgie ambulatoire survient dans $0,2 \%$ des cas et varie selon le type de service. Le nombre de patients non accompagnés est plus élevé que celui des patients qui savent avant l'opération qu'ils se présenteront seuls.

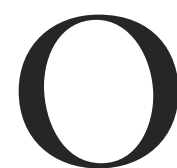
NE of the discharge criteria recommended by the American Society of Anesthesiologists (ASA) is to have a responsible individual accompany the patient home (an escort). ${ }^{1}$ It is believed that having an escort reduces adverse outcomes, increases patient comfort and satisfaction, and should be mandatory. However, the literature has been silent as to whether the presence of an escort to accompany the patient home after discharge is associated with a decrease in postdischarge complications or other adverse outcomes. Furthermore, no study has focused on the issue of the ambulatory surgical patient without an escort.

The purpose of this study was to identify the characteristics of ambulatory surgical patients without an escort, and to compare their outcomes with a matched control group of patients with an escort.

From the Departments of Anesthesia, ${ }^{*}$ and Nursing, $†$ Toronto Western Hospital, University Health Network, University of Toronto, Toronto, Ontario, Canada. Address correspondence to: Dr. Frances Chung, Department of Anesthesia, Toronto Western Hospital, 399 Bathurst St., EC 2-046,

Toronto, Ontario M5T 2S8, Canada. Phone: 416-603-5118; Fax: 416-603-6494; E-mail: frances.chung@uhn.on.ca Accepted for publication May 25, 2005. Revision accepted August 9, 2005. 


\section{Methods}

Following Institutional Research Board approval, we prospectively collected data of consecutive patients with no escort in the Ambulatory Surgery Unit of the Toronto Western Hospital for a 38-month period (2001-2004). "No escort" is defined as not having a responsible individual to accompany the patient home. As this was an observational study, the Ethics Committee did not require written informed consent from the patients before data collection. The anesthesiologists and the surgeons were not aware of the prospective clinical collection of data on no escort in ambulatory surgical patients. Only the nurses in the ambulatory surgical unit were aware of the clinical study.

Demographic data including age, ASA classification, type of anesthesia, type of operation, and the reason for no escort was collected on the "no escort" patients. Following surgery and recovery, should a patient not have an escort, the surgeon and anesthesiologist were informed, and an effort to find an inpatient bed was made. If hospital admission was not possible, the anesthesiologist explained possible complications to the patient. It was stressed that if the patient had to travel alone or were home alone, he or she might not be able to initiate contact with the emergency medical agencies when required. The patient was asked to sign against medical advice to be discharged home.

It is a routine practice in our institution to telephone all ambulatory surgical patients $24 \mathrm{hr}$ postoperatively. Questions regarding pain, bleeding, etc. were asked during a phone call and recorded. All patients who had surgery and were discharged with no escort were also contacted $24 \mathrm{hr}$ later by phone. Information including travel distance, whether an adult stayed with the patient overnight, whether the patient had to take care of minors at night, and complications, such as severe pain or bleeding were documented.

For comparative purposes, a case control group was selected. To select control cases for our clinical report, we randomly chose patients who had surgery during the same time period to match the patients with an escort. Only patients who had an escort, underwent similar procedures at the same period, and well matched for age, sex, ASA and type of anesthesia with the patients with an escort, were chosen to be control cases. Data of hospital readmission and emergency visits within 30 days were collected by chart review for both groups of patients, with and without an escort.

Statistical analysis was performed with Chi-squares and Fisher exact test as appropriate. A $P$ value $<0.05$ was considered statistically significant. Post hoc power
TABLE I Characteristics of patient without an escort and matched group

\begin{tabular}{llll}
\hline & $\begin{array}{l}\text { Patient with } \\
\text { no escort } \\
(n=55)\end{array}$ & $\begin{array}{l}\text { Patient with } \\
\text { an escort } \\
(n=55)\end{array}$ & \\
\hline Sex ratio (male:female) & $23: 32$ & $23: 32$ & NS \\
$\begin{array}{l}\text { Age mean } \\
\text { Age (range) }\end{array}$ & 39.78 & 41.47 & NS \\
$\begin{array}{l}\text { Type of operation } \\
\text { D\&C }\end{array}$ & $18-72$ & $20-75$ & NS \\
Orthopedic/hand/plastic & 18 & & \\
Ophthalmology & 5 & 18 & NS \\
Chronic pain & 3 & 5 & NS \\
General surgery & 2 & 3 & NS \\
Biopsy & 1 & 2 & NS \\
Urology & 1 & 1 & NS \\
ASA & & 1 & NS \\
I & 30 & 27 & NS \\
II & 17 & 21 & NS \\
III & 7 & 7 & NS \\
IV & 1 & 0 & NS \\
Type of anesthesia & & & \\
General anesthesia & 35 & 35 & NS \\
Regional \pm sedation & 8 & 9 & NS \\
Monitored anesthesia care & 12 & 11 & NS \\
Total & 55 & 55 & NS \\
\hline ASA Am
\end{tabular}

ASA $=$ American Society of Anesthesiologists, D\&C $=$ dilatation and curettage; NS = not significant. Note: this table includes only data from the patients who underwent surgery without an escort $(n=55)$. The patients whose surgery was cancelled $(n=5)$ due to no escort were not included.

calculations were carried out using the formulas in Devore (1999) for power and sample size for comparing two binomial proportions. ${ }^{2}$

\section{Results}

The demographic characteristics of patients without an escort and the matched control group of patients are summarized in Table I. No significant differences were observed regarding age, sex, ASA classification, type of surgery, and type of anesthesia between the two groups.

Over a 38-month study period, the incidence of ambulatory patients without an escort was $0.2 \%$ $(60 / 28,391)$. The highest incidence of patients without an escort was observed in gynecology patients undergoing termination of pregnancy (1.2\%). Table II summarizes the incidence of no escort patients according to type of surgery.

Our data identified two groups of patients without an escort, patients $(n=24)$ known not to have an escort preoperatively (known no escort) and patients $(n=36)$ who claimed that they had an escort, but the escort did not show (no show escort). The demographic characteristics of these two groups of patients 
TABLE II Incidence of no escort patients during a 38month period according to service

\begin{tabular}{llll}
\hline & $\begin{array}{l}\text { No escort } \\
\text { patients }(n)\end{array}$ & $\begin{array}{l}\text { Patients } \\
\text { operated }(n)\end{array}$ & $\begin{array}{l}\text { Incidence } \\
(\%)\end{array}$ \\
\hline D\&C & 27 & 2,291 & 1.17 \\
Orthopedic/hand/plastic & 20 & 6,956 & 0.29 \\
Ophthalmology & 5 & 11,864 & 0.04 \\
Chronic pain & 3 & 635 & 0.47 \\
General surgery & 2 & 1,773 & 0.11 \\
Biopsy & 1 & 1,418 & 0.07 \\
Urology & 2 & 606 & 0.33 \\
Other & 0 & 2,848 & 0 \\
Total & 60 & 28,391 & 0.21 \\
\hline
\end{tabular}

$\mathrm{D} \& \mathrm{C}=$ dilatation and curettage.

TABLE III Demographic data of patients who underwent surgery without an escort

\begin{tabular}{lll}
\hline & $\begin{array}{l}\text { "No show” escort } \\
(n=36) \\
\text { Patients (n, \%) }\end{array}$ & $\begin{array}{l}\text { Known no escort } \\
(n=19) \\
\text { Patients (n, \%) }\end{array}$ \\
\hline $\begin{array}{ll}\text { Sex } \\
\text { Female }\end{array}$ & $21(58)$ & $11(58)$ \\
Male & $15(42)$ & $8(42)$ \\
Type of surgery & $19(52.8)$ & $6(31.6)$ \\
D\&C & $11(30.6)$ & $7(36.8)$ \\
Plastic/hand/orth & $1(2.8)$ & $0(0)$ \\
Urology & $2(5.6)$ & $1(5.3)$ \\
Chronic pain & $2(5.6)$ & $1(5.3)$ \\
General & $1(2.8)$ & $4(21.1)$ \\
Ophthalmology & & \\
ASA & $22(61)$ & $8(42)$ \\
I & $8(22)$ & $9(47)$ \\
II & $5(14)$ & $2(11)$ \\
III & $1(3)$ & $0(0)$ \\
IV & & \\
Type of anesthesia & $25(69)$ & $8(42)$ \\
General anesthesia & $6(17)$ & $3(16)$ \\
Regional \pm sedation & $5(14)$ & $8(42)$ \\
Monitored anesthesia care & $5(14)$
\end{tabular}

$\mathrm{D} \& \mathrm{C}=$ dilatation and curettage.

who underwent surgery are summarized in Table III. The group of patients with "known no escort" underwent more ophthalmic surgery and more monitored anesthesia care, compared to the group of "no show escort" patients.

In the "known no escort" group, 22 patients had knowledge of the need for an escort; two patients claimed no such knowledge. Five of these patients had their surgery cancelled because of no escort. Four patients were admitted electively after their surgery. Fifteen of these patients had their surgery performed, and were discharged.

Telephone follow-up was done within $24 \mathrm{hr}$ after surgery. Of the 51 patients who had surgery and were discharged home without an escort, we were able to contact 39 patients $(76.5 \%)$. Eleven patients who had termination of pregnancy and one heart biopsy patient could not be contacted. The living arrangements of these patients are summarized in Table IV.

Information regarding severe pain and bleeding was collected by telephone follow-up. There was no significant difference in bleeding $24 \mathrm{hr}$ postoperatively between the no escort group and the matched control group. The no escort group had a significantly higher incidence of severe pain $v s$ the matched control group $(P<0.02)$.

The intended outcomes in no escort group and matched control group are summarized in Table V. In the no escort group, four patients had elective unanticipated admission due to no escort. Two patients were readmitted into hospital within 30 days: one for infection, and one for repeated surgery for retinal detachment. One patient visited the emergency room because of bleeding, while in the matched control group, there was no unanticipated admission or readmission. Only one patient had a visit to emergency because of pain and bleeding after surgery. There were no differences in the rates of unanticipated admission, emergency room visits, and readmission within 30 days between the group of patients with and without an escort.

\section{Discussion}

Recovery of patients after anesthesia has three different phases. ${ }^{3,4}$ Early recovery occurs when a patient emerges from anesthesia; intermediate recovery occurs when a patient has satisfied criteria for discharge; and late recovery occurs when the patient has returned to their preoperative physiological state. Following surgery, patients are discharged home when they have satisfied discharge criteria, ${ }^{5,6}$ but not necessarily back to their preoperative physiological state. Various studies have shown that there is significant psychomotor and cognitive impairment after anesthesia. ${ }^{7-9}$ Therefore, it is recommended that patients scheduled for ambulatory surgery have a responsible adult to accompany them home and stay with them overnight. Escorts can ensure that the patient safely arrives home, and should assist the patient with minor postoperative symptoms such as pain, nausea, vomiting. In case of an emergency, suitable medical help can be easily reached. ${ }^{10-12}$

Our results showed that there are no statistically significant differences in the rates of unanticipated admission, emergency visit and readmission within 30 days when comparing a group of patients with, and without an escort. Those four unanticipated admission 
TABLE IV Living arrangements of patients discharged without an escort

\begin{tabular}{lll}
\hline & No. of patients $(n=39)$ & Incidence (\%) \\
\hline Live far from hospital (> two hours driving distance) & 4 & 10.3 \\
No responsible adult at home with patient & 28.2 & 3 \\
Solely responsible for minors during first night after surgery & 7.7 \\
\hline
\end{tabular}

TABLE V Outcome of patients with no escort and the matched control group

\begin{tabular}{llc}
\hline & Patient with no escort & Patient with an escort \\
\hline Unanticipated admission & $4 / 55$ & $0 / 55$ \\
Readmitted within one month & $2 / 55$ & $0 / 55$ \\
Emergency visit & $1 / 55$ & NS \\
\hline
\end{tabular}

patients had elective admission due to no escort. In order to determine whether this study had adequate power to detect the observed effects for all adverse outcomes, we conducted post hoc power analysis based on this observational study. We calculated the power with a type I error rate of 0.05 sample size and the observed event rates obtained from this study. Unanticipated admission, readmission within 30 days and emergency visits are our main concern: the post hoc powers are $65 \%, 42 \%$ and $5 \%$ respectively.

In a recent survey of anesthesiologists, $11.2 \%$ were willing to anesthetize patients without an escort. ${ }^{13}$ In this study, 19 patients were known not to have an escort, but surgeries were still performed. The results of this study show that these patients had a higher percentage of ophthalmic surgery and monitored anesthesia care vs the group of patients with "no show escort". Some anesthesiologists may believe that patients who do not receive general anesthesia may be able to go home without an escort. At present, there is insufficient evidence to support this belief.

Another interesting finding from our data is that over half of the no escort patients claimed that they had an escort, but the escort did not show after surgery. Some of these patients might not have an escort arrangement preoperatively; failure to show might be due to other reasons. These are unpredictable and hard to avoid. We should encourage these patients to find an alternative escort. If none is available, these patients can be admitted into hospital if possible. Some hospitals have the policy of a 'hotel bed' where patients can stay in the hospital overnight without necessarily having nursing staff attend to their care. These patients do, however, have easy access to emergency health care.
A survey of 750 ambulatory surgical patients showed that $4 \%$ of ambulatory surgical patients had no responsible adult staying with them overnight, ${ }^{14}$ while in this report, a higher percentage $(28.2 \%)$ of patients who went home without an escort had no responsible adult staying with them overnight. Also, some of the no escort patients had to travel over two hours after their surgery.

There are some limitations to the study. The data were collected from one tertiary care institution. This may limit the 'generalizability' to other centres, including community hospitals. Due to the small sample size of no escort patients, and the fact that infrequent but catastrophic events could occur, we cannot formulate a conclusion regarding adverse outcomes of patients secondary to 'no escort'.

To summarize, the absence of an escort in ambulatory surgical patients is a recurrent problem with an incidence of $0.2 \%$. The number of patients with no show escort is much higher than the number of patients with known no escort preoperatively. A high percentage $(28.2 \%)$ of patients who went home without an escort had no responsible adult staying with them overnight. It is possible that patients may have increased adverse events due to no escort. A large multicentre study is needed to further clarify this problem of no escort in ambulatory surgical patients.

\section{References}

1 American Society of Anesthesiologists Task Force on Postanesthetic Care. Practice guidelines for postanesthetic care. A report by the American Society of Anesthesiologists Task Force on Postanesthetic Care. Anesthesiology 2002; 96: 742-52.

2 Devore JL. Probability and Statistics for Engineering 
and the Sciences, 5th ed. California: Duxbury Inc.; 1999: 387-8.

3 McGrath B, Chung F. Postoperative recovery and discharge. Anesthesiol Clin North America 2003; 21 : 367-86.

4 Chung F, Chan VW, Ong D. A post-anesthetic discharge scoring system for home readiness after ambulatory surgery. J Clin Anesth 1995; 7: 500-6.

5 Kortilla $K$. Recovery and home readiness after anesthesia for ambulatory surgery. Semin Anaesth 1990; 9: 182-9.

6 Marshall SI, Chung F. Discharge criteria and complications after ambulatory surgery. Anesth Analg 1999; 88: 508-17.

7 Apfelbaum JL, Lichtor JL, Lane BS, Coalson DW, Korttila KT. Awakening, clinical recovery, and psychomotor effects after desflurane and propofol anesthesia. Anesth Analg 1996; 83: 721-5.

8 Grant SA, Murdoch J, Millar K, Kenny GN. Blood propofol concentration and psychomotor effects on driving skills. Br J Anaesth 2000; 85: 396-400.

9 Sinclair DR, Chung F, Smiley A. General anesthesia does not impair simulator driving skills in volunteers in the immediate recovery period - a pilot study. Can J Anesth 2003; 50: 238-45.

10 Wolff J, Dunnihoo DR. A freestanding ambulatory surgical unit: a success or failure? Am J Obstet Gynecol 1982; 143: 270-6.

11 Rudkin GE, Osborne GA, Doyle CE. Assessment and selection of patients for day surgery in a public hospital. Med J Aust 1993; 158: 308-12.

12 Lieber CP, Seinige UL, Sataloff DM. Choosing the site of surgery. An overview of ambulatory surgery in geriatric patients. Clin Geriatr Med 1990; 6: 493-7.

13 Friedman Z, Chung F, Wong DT. Ambulatory surgery adult patient selection criteria - a survey of Canadian anesthesiologists. Can J Anesth 2004; 51: 437-43.

14 Correa R, Menezes RB, Wong J, Yogendran S, Jenkins $K$, Chung $F$. Compliance with postoperative instructions: a telephone survey of 750 day surgery patients. Anaesthesia 2001; 56: 481-4. 